\title{
PHOTOVOLTAICS:
}

\section{TOWARDS ULTIMATE PERFORMANCES}

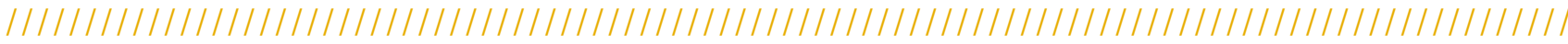

\section{J.F. GUILLEMOLES}

CNRS, UMR IPVF 9006, Institut Photovoltaique d'île de France, École Polytechnique IP Paris, PSL Chimie ParisTech, IPVF SAS, 91120 Palaiseau, France

*jean-francois.guillemoles@cnrs.fr

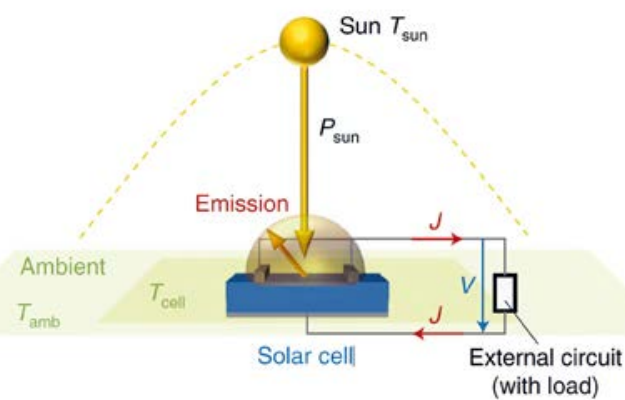

Photovoltaic conversion has made impressive progress since its discovery, but as will be discussed here, much can still be done. It is of interest to investigate what are the intrinsic limits of the technology. We call here ultimate performance those that could be limited only by intrinsic properties of the devices. This point will be discussed along three lines: ultimate limits for energy conversion, ultimate limits for material usage and ultimate limits for device stability.

This is an Open Access article distributed under the terms of the Creative Commons Attribution License (http://creativecommons.org/licenses/by/4.0), which permits unrestricted use, distribution, and reproduction in any medium, provided the original work is properly cited.

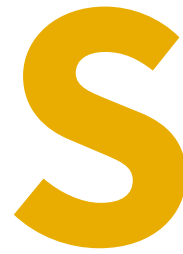
olar energy, because of its abundance, availability everywhere, and innocuity, is considered among the main sources of renewable energy and an important pillar in the current energy transition. Indeed, Photovoltaic effect, discovered in 1839 by E. Becquerel [1], has made impressive progress from being a laboratory curiosity to contributing about $3 \%$ of the world electricity production in 2021. Progress have been made on conversion efficiency (now commercially above $20 \%$ ),

Figure 1: Solar cell operation (from [2]). (a) A solar cell absorbs the light from the sun and emits electrons in the external circuit, the load, while excess energy is rejected in the ambient. (b) Energy diagram of an operating solar cell: an absorbed photon promotes an electron from valence to conduction band (A), the excess energy is dissipated (B) and some potential energy, that depends on the operating voltage $\mathrm{V}$, can be collected (C).

a

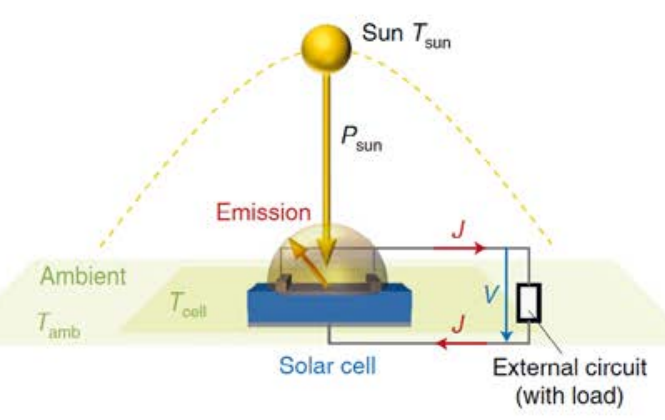

b

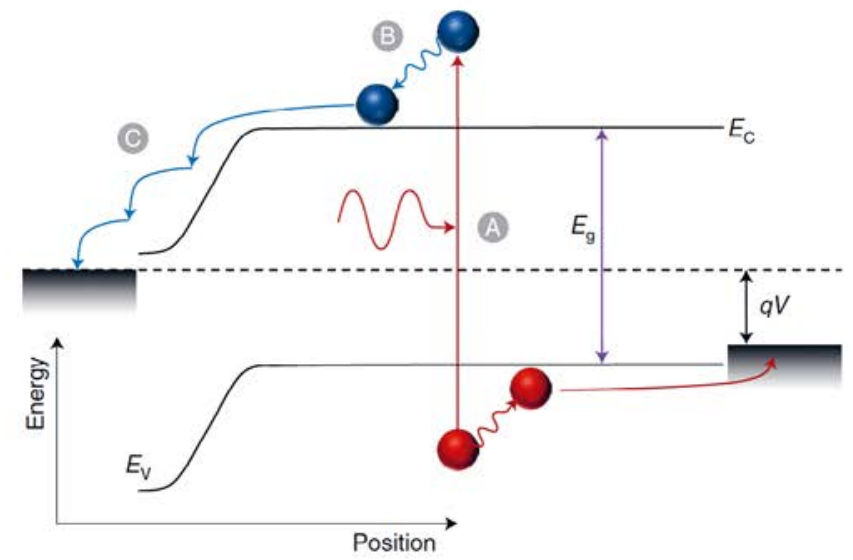


semiconductor, in a process that promass production (now close to TW capacity installed), cost (one of the cheapest energy in sunny places, with LCOE near $1 \mathrm{ct} / \mathrm{kwh}$ ), reliability (20 to 30 years' warranty on lifetime), energy return on energy invested (about 20 times), etc...

That is not to say there are no impediments to large scale use of solar energy. Some preeminent issues today: the supply is variable and with the current technology, it would require large amounts of materials. These issues are subject to intense research, and could be solved. I will not discuss them further here, but will rather discuss another question: can we define some intrinsic limitations to photovoltaic conversion, and if so, which? By intrinsic, I mean that are deeply rooted in physics, and would not depend on the specifics of the operation conditions.

I will shortly discuss below three such limits. First, there is certainly a limit to energy conversion efficiency, how far is the state of the art from that point? This question has a bearing on cost and on how cheap solar electricity could ultimately become, but also on the environmental footprint of the technology and on the opening of a larger range of applications. It depends on the quality of the semiconductors used, but also on the architecture of the devices, ... Second, there is a limit on the amount of material that has to be mobilized to make efficient devices. This question may have a bearing on cost (if expensive materials are used), footprint (if scarce materials are used). As will be seen, some device concepts may require a tiny amount of active materials only.

Third, as p/n junctions, widely used for this application, are kinetically stable, but not intrinsically stable, it is an open question whether that would set a limitation on the time of use of solar cells. This question could also have a bearing on ultimate cost and footprint of the technology.

\section{LIMITS TO EFFICIENCY}

As surprising as it is, efficiency limits of photovoltaic conversion are ultimately set by optical properties. As there is an energy threshold (or gap energy, Eg, Fig. 1b) for light absorption in a motes an electron from the valence band into the conduction band, only the fraction of the incoming photons having an energy above Eg (more precisely the fraction that is effectively absorbed, determined by the optical properties $(\mathrm{n}, \mathrm{k})$ of the device) can be used: it determines the photogenerated current. Most of the carriers' excess energy, above threshold, is quickly degraded into heat and given away to the lattice by fast, activationless processes, till carriers have an average kinetic energy on the order of $k T, \mathrm{k}$ being the Boltzmann constant, so that the energy per photogenerated carrier is slightly above Eg. Therefore, the energy threshold limits at the same time the amount of photons that can be absorbed (and therefore the photocurrent), and the energy available per carrier (see Fig. 2a): this gives an optimal value for a diode with Eg about $1 \mathrm{eV}$ and $44 \%$ efficiency, an efficiency that is independent of source and sink temperature, and of illumination intensity, therefore a crude model. A much improved model was proposed by Shockley and Queisser [3], that considered also the impact of temperature, illumination intensity and operating bias on the efficiency.

In simple terms, a solar cell works by absorbing solar photons that generate excited electrons. Some of these would be eventually collected at one electrode and after having performed some work in the external circuit, would be reinjected at the other electrode (see Fig. 1).

In its simplest form, a photovoltaic device is composed of a semiconductor and two selective contacts, one for holes (exchanging carriers with the valence band mostly), and the other for electrons holes (exchanging carriers with the conduction band mostly): a typical example of such a device is a semiconductor $\mathrm{p} / \mathrm{n}$ junction. The efficiency of any such device depends on its operation point, that is light intensity, temperature and terminal voltage (or equivalently, load impedance). Let's start with the temperature: like any conversion device, a solar cell is subject to the laws of thermodynamics. A solar cell is an engine that converts a heat flux coming from the sun (almost a black-body source at $5800 \mathrm{~K}$ )

\section{THE ID281 SERIES}
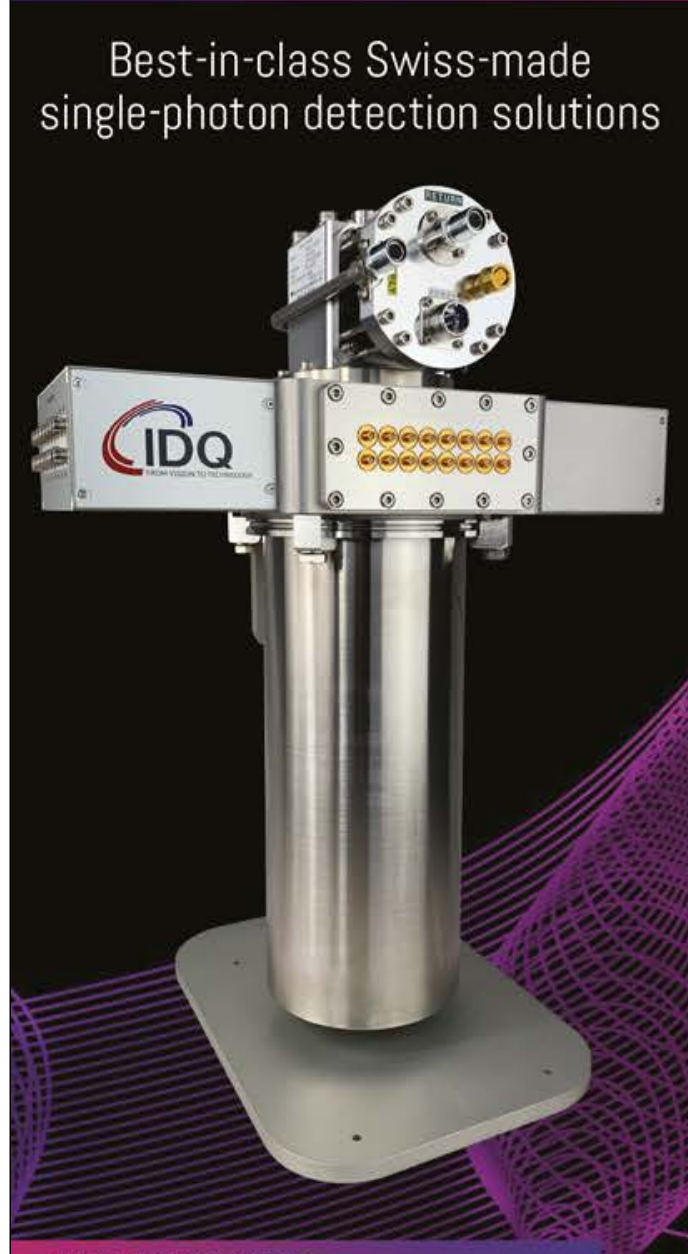

SEE EVERYTHING

Mix and match up to 16 single-photon

broad band detector channels, with

unparalleled system efficiency

\section{SEE CLEARLY}

Ultra-precise timing jitter and superb

noise performance

\section{MAKE EVERY PHOTON COUNT}

Count more photons with real-time

photon-number-resolving solutions

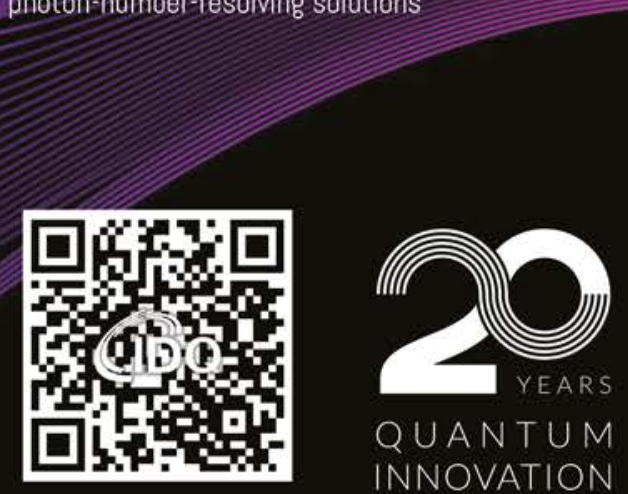


and dumped at ambient temperature (the cold sink) into electrical work (Fig. 1a). Put in these terms it should be bounded by the Carnot efficiency (about 95\%). Clearly, when the cold sink temperature rises, the efficiency is expected to decrease. Indeed, a complete analysis of a solar cell yields a Carnot efficiency factor in its terminal open circuit voltage.

Turning now to the terminal voltage: under forward bias, electrons are extracted through the electron selective contact but in doing so they work against the electric bias (conversely, when the diode is reverse biased, photogenerated carriers are quickly extracted from the solar cell, but at the price of an extra work). This work is then available in the load (driving a motor, the charge of a battery, ....). The fact that photogenerated carriers have to go against an electric bias to be extracted increases the chances that they relax to their fundamental state (a process called recombination): as the forward voltage $\mathrm{V}$ increases, so does the available potential energy per extracted carrier, while the flux of carriers (intensity I) goes down. At zero bias, the current (so called short circuit current) is close to the absorbed photon flux in state of the art devices. For some terminal voltage (called open circuit voltage, Voc) the output current is zero (Fig. 2b). Now, because the power output of the device is $\mathrm{P}=\mathrm{I} . \mathrm{V}$, it will reach a maximum between zero bias and Voc: this is the optimal working point of the device (for a given temperature and light flux).

Finally, the efficiency depends also on the light intensity. At high light intensity, the concentration of photogenerated carriers is larger (for a given bias and temperature), and therefore the cell voltage is larger. Because the work delivered per collected carrier depends on the carrier concentration, light concentration is beneficial.

Because of these effects, at $300 \mathrm{~K}$ working temperature, and standard solar illumination, the optimal diode would yield an efficiency of 33\%, with about $30 \%$ of non-absorbed light, the rest being lost in heat during the initial thermalisation process or during the collection process. A single semiconductor cannot optimally make use of the broad solar spectrum, but it can be optimized for a narrow band of the solar spectrum. A collection of semiconductors with adjusted band gaps can be used to cover more optimally the conversion of the full solar. This is the main technological route to achieve high efficiencies, as the bottleneck is essentially engineering. In 2021, such devices, called multijunctions, using up to 6 diodes (each with adjusted Eg), have achieved conversion efficiencies of $47 \%$ [4] which is quite an impressive achievement already. Pushed to the limit, with essentially a diode for each narrow wavelength range,

Figure 2: Shockley Queisser limit (from [2]). a) fraction of power optimally converted as a function of band gap of an idealized solar cell (Shockley-Queisser model). Non-absorbed and thermalized power are explained in text. There is an irreductible fraction of carriers that recombines emitting photons before being collected (b), and a usually larger fraction still that recombines by non-radiative processes, thereby reducing available current, while the entropy produced, called here isothermal losses, during the recombination processes also reduces the available voltage (details in [2b]).
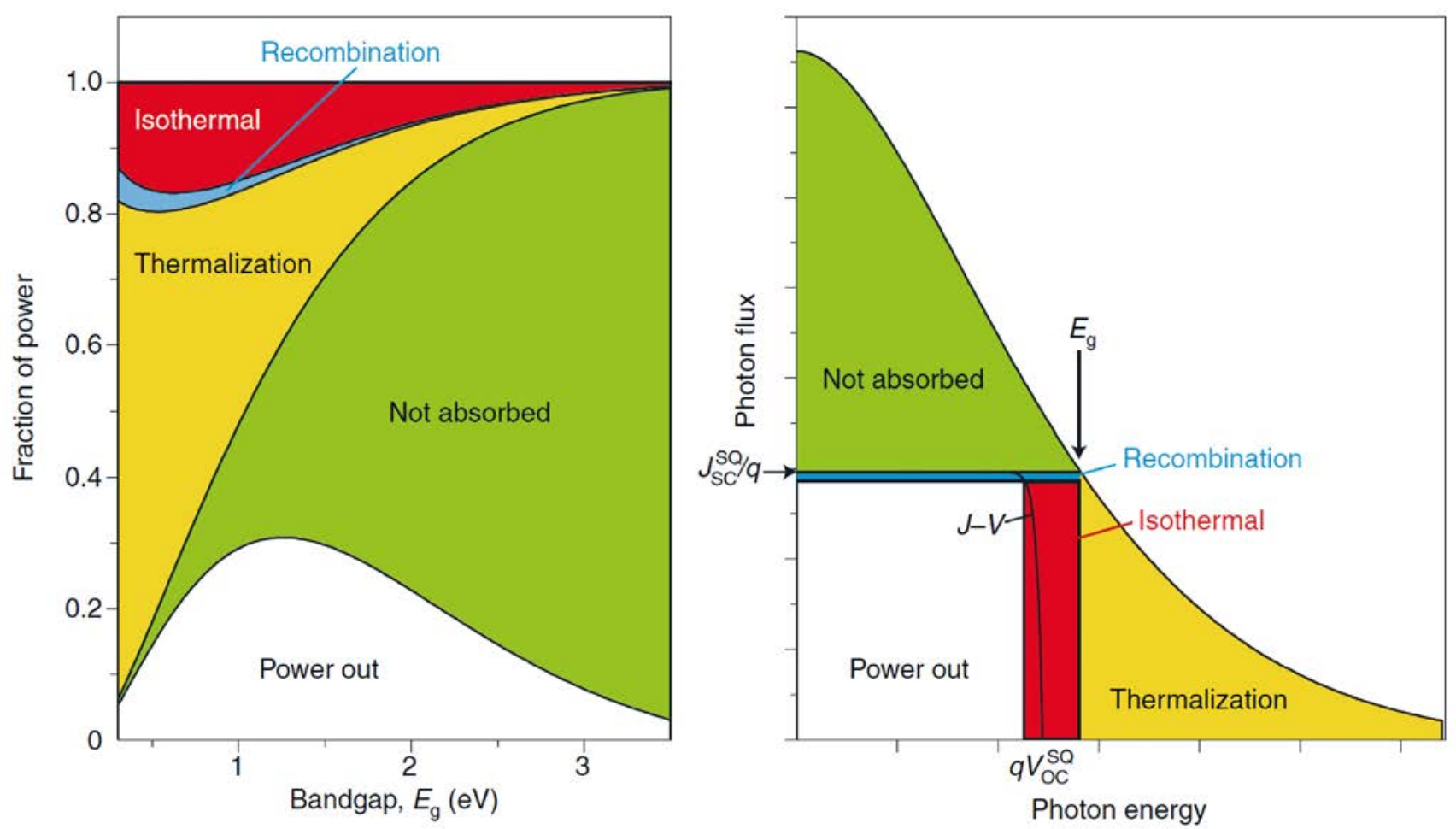


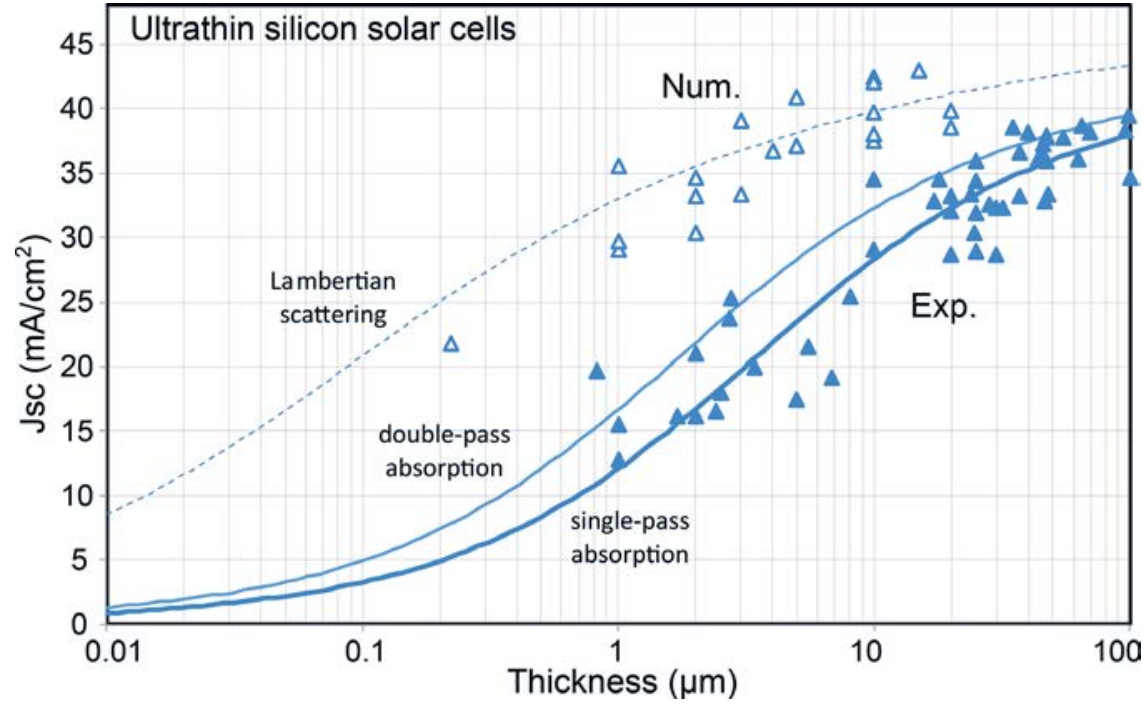

Figure 3: Dependence on thickness of the maximal photogenerated current, experimentally (filled triangles) and theoretically (empty triangles) for Si cells. The lower curve is obtained by absorption from Beer's law, and the top curve from Lambertian light trapping. From [6].

such devices could reach the ultimate photovoltaic efficiencies of $68 \%$ under one sun of $87 \%$ under maximal solar concentration achieved when the solar cell "sees" the sun over a $4 \pi$ solid angle).

A variety of device architectures have been proposed and tested. As effective as it is, these devices require many technological steps, and are very complex and expensive to make, especially for the most efficient designs. Other research directions are being pursued to reach similar efficiencies as the multijunctions, but with much simpler device architectures. For instance, hot carrier solar cells would work at the same time as a photovoltaic and a thermoelectric engine [5]. First proofs of principle have been made but there are still many issues on the way related to photonics, and materials and device engineering.

\section{LIMITS TO MATERIAL USAGE}

Even if solar cells were approaching their limiting efficiency, current technologies would require large amounts of active materials (semiconductors), often in a highly pure form. But yet another expectation on solar cells is that they should be produced with minimal environmental impact.
Using solar concentration (the limit being 45000 times standard illumination, when the solar cell "sees" the sun over a $4 \pi$ solid angle), the amount of active material needed could be reduced by the concentration factor. A less recognized way of reducing material use is to reduce the thickness of solar cells. Using photonic concepts, again ultimately, a factor in thickness reduction close to 2 orders of magnitude as compared to standard technology could be gained. For instance, Silicon solar cells, today $200 \mu \mathrm{m}$ thick, could in principle be a couple of $\mu \mathrm{m}$ thick (figure 2), this direction of research is only in its infancy, but is very promising. As this approach has already been developed [6], the details will not be repeated here.

Taken together, concentration and thinning enable a conversion process by a very small amount of active materials, ultimately down to few grams for GW production capacity . Of course, to this reduced amount of active materials, of metals, for the electrical contacts, of optical materials to collect the solar illumination and channel it to the device, and structural materials for mechanical and environmental integrity). one needs to add a significant quantity
2BLighting Technologies

\section{High performance and reliable} fiber optic assemblies

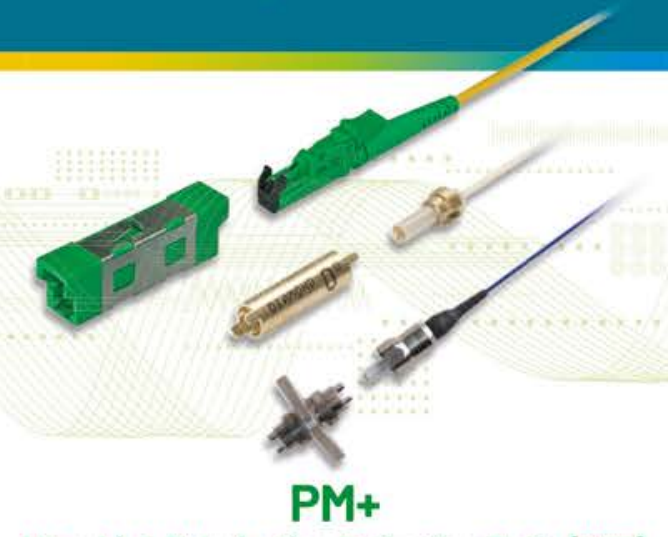

Ultra high Polarization Extinction Ratio (PER)

- Up to $+4 \mathrm{~dB}$ higher PER

-State-of-the-art insertion Loss (IL) and Return Loss (RL) values

- Best connector type and tolerance $\mathrm{E}-2000^{\circ}$, DMI, Mini AVIM $^{\circ}$ and Micro AVIM ${ }^{\circ}$

- Available on homologated fibres and cables

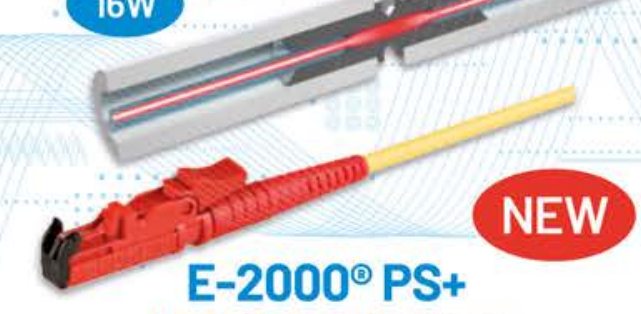

Contact expanded beam - Low loss

- Interlock solution optional

. 1310-1550nm or 980-1060nm

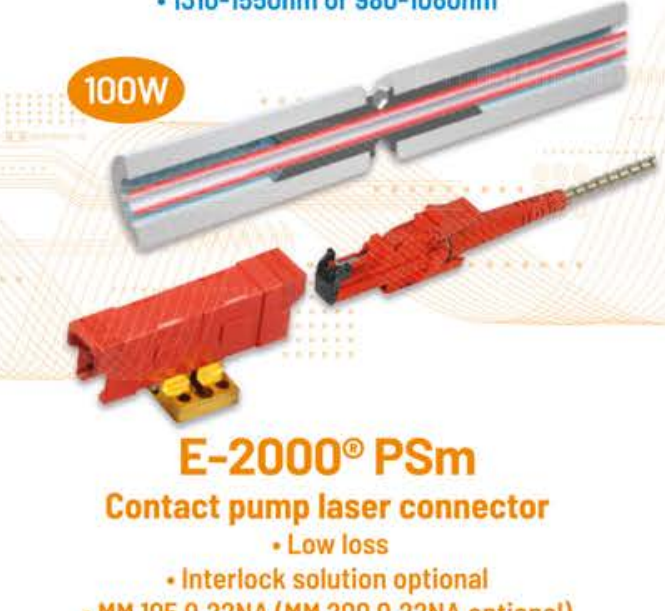

- MM 105 0.22NA (MM 200 0.22NA optional)

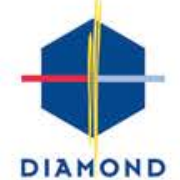

www.2blighting.fr

info@2blighting.com

$+33164592130$ 


\section{LIMITS TO DURABILITY}

Another aspect of the operation of a solar cell, is that it should produce with minimal degradation during decades, in spite of environmental aggressions.

Solar cells do wear out in outdoor conditions, especially as thermal cycling may induce cracks, as water could ingress in the modules and corrode the contact, encapsulation polymers could degrade and absorb part of the incoming light, ..., but some devices have been working since their fabrication, decades ago, without trouble. Light absorption does produce excited electrons, and there is a small, but non-zero possibility that defects will be created in the absorbing material, reducing efficiency over time. Moreover, $\mathrm{p} / \mathrm{n}$ junctions are intrinsically unstable since they result from a gradient in doping concentration that are subject to both Fick's law and the built in electric field as forces opposing the existence of the gradient. This causes a fundamentally limited intrinsic device stability [9], especially in the case of ultrathin devices, where this effect would be exacerbated.

Presently, device stability is ensured either by reducing the kinetics of damage, or by halting damage, possibly by selecting materials with high activation barriers to damage. A potentially more robust way of achieving the same goal would be to design self-healing devices, and a proposal to achieve that is to design thermodynamically stable devices.

Interestingly, the advent of intrinsically stable $\mathrm{p} / \mathrm{n}$ junctions is possible as thermodynamically stable homo- and hetero-junctions have been discovered. An example of this is given by silver-doped MCT (Mercury Cadmium Telluride), where homojunctions, smeared out by application of a strong field or high temperature would spontaneously reform [8]. This system is an example of a dynamically stable device (likely the result of a spinodal decomposition between $\mathrm{n}$ and $\mathrm{p}$ regions), as opposed to say, silicon homojunctions, that are kinetically stable (frozen non-equilibrium).

Other semiconductors appear to have an intrinsic defect population that, at equilibrium, is optimal or close to optimal for PV conversion, and when perturbed, appears to spontaneously return to their functioning state. This seems to be the case of CIGS [7], and could be the case of the quickly emerging halide perovskites. This property does not only allow the device to work for long, but also enables the device to operate in specific environments, like space, where end of life is determined by radiation hardness.

Even if possibilities appear to emerge in the far distance, it should be reckoned that today's solar cell are not limited in use by their intrinsic stability but rather by the impact of environment mostly on the structural elements that protect the active material.

\section{CONCLUSIONS}

When looking closely to the physics of solar cells, quite general conclusions could be made on intrinsic possibilities offered by this category of energy conversion devices, and what could be achieved ultimately. Specifically, the ultimate conversion device could be extremely efficient (over 80\%), using very little of hardto-produce active material (few g/ $\mathrm{GW}$ ) and could be used for indefinitely long time and in a variety of environments, thanks to self-healing properties that could be imparted to the device.

As for today, as these fundamentals topics are an active research topic, other more pressing issues are actually impeding today the use of Photovoltaic devices, such as the ability to be integrated in diversified usage, which is also subject to intense R\&D. Nevertheless, the conclusion of the above is that the ultimate cost and footprint of the technology has a very low limit, and therefore is likely to further progress for quite some time in the future.

REFERENCES

[1] A. Becquerel, Comptes rendus 9, 561-567 (1839)

[2]J.-F. Guillemoles, T. Kirchartz, D. Cahen and U. Rau, Nat. Photonics 13, 501-505 (2019)

[2b] J.F. Guillemoles, Thomas Kirchartz, David Cahen and Uwe Rau, Nat. Photonics 15, 165-166 (2021)

[3] William Shockley and Hans J. Queisser, J. Appl. Phys. 32, 510-519 (1961)

[4] M. A. Green, E. D. Dunlop, J. Hohl-Ebinger et al., Prog Photovolt Res Appl. 29, 657-667 (2021)

[5] D. T. Nguyen, L. Lombez, F. Gibelli et al., Nat. Energy 3, 236-242 (2018)

[6] I. Massiot, A. Cattoni, S. Collin, Nat. Energy 5, 959-972 (2020);

cellules solaires ultrafines, Photoniques 102, Mai-Juin 2020,

https://doi.org/10.1051/photon/202010244

[7] J.F. Guillemoles, U. Rau, L. Kronik, H.W. Schock, and D. Cahen, Adv. Mater. 11, 957-961 (1999)

[8] I. Lyubomirsky, V. Lyakhovitskaya, J.F. Guillemoles et al., J. of Crystal Growth 161, 90-93 (1996)

[9] J.-F. Guillemoles, I. Lyubomirsky, I. Riess, and D. Cahen, J. Phys. Chem. 99 , 14486-14493 (1995) 